\title{
Generalized surface momentum balances for the analysis of surface dilatational data
}

\section{Journal Article}

\section{Author(s):}

Sagis, L. M. C.

Publication date:

2013-05

Permanent link:

https://doi.org/10.3929/ethz-b-000069455

Rights / license:

In Copyright - Non-Commercial Use Permitted

Originally published in:

The European Physical Journal Special Topics 222(1), https://doi.org/10.1140/epjst/e2013-01823-2 


\title{
Generalized surface momentum balances for the analysis of surface dilatational data
}

\author{
L.M.C. Sagis ${ }^{1,2, a}$ \\ 1 Food Physics Group, Wageningen University, Bomenweg 2, 6703 HD Wageningen, \\ The Netherlands \\ 2 Polymer Physics, Department of Materials, ETH Zurich, Wolfgang-Pauli-Str. 10, \\ 8093 Zurich, Switzerland
}

Received 16 April 2013 / Received in final form 23 April 2013

Published online 17 June 2013

\begin{abstract}
Dilatational rheological properties of interfaces are often determined using drop tensiometers, in which the interface of the droplet is subjected to oscillatory area changes. A dynamic surface tension is determined either by image analysis of the droplet profile or by measuring the capillary pressure. Both analysis modes tend to use the Young-Laplace equation for determining the dynamic surface tension. For complex fluid-fluid interfaces there is experimental evidence that this equation does not describe the response of the interface to deformations adequately. Generalizations of this equation are available, and in this comment we will discuss these generalizations, and the conditions for which they reduce to the Young-Laplace equation.
\end{abstract}

\section{Introduction}

In oscillating droplet (OBM) and bubble pressure tensiometry (BPT) experiments data for the dilatational modulus are in general obtained by subjecting an interface to oscillatory area changes, and analyzing either the droplet profile or the pressure difference over the interface using the Young-Laplace equation (see for example the review by Javadi et al. elsewhere in this issue [1]). The Young-Laplace equation (sometimes also referred to as Gauss-Laplace equation or simply Laplace equation) is given by

$$
P^{(2)}-P^{(1)}=2 \gamma H
$$

where $P^{(2)}$ is the pressure in the interior of the droplet, $P^{(1)}$ is the pressure in the outer phase, $\gamma$ is the surface tension, and $H$ is the curvature of the interface $(=1 / R$ for spherical droplets, where $R$ is the droplet radius). The analysis of tensiometry experiments with this equation tends to work well for interfaces stabilized by low molecular weight surfactants that do not form complex mesophases after adsorption at the interface. However, surface active components such as colloidal particles, polymers, and proteins may exhibit strong in-plane interactions after adsorption, which can lead to the formation of two dimensional gel, glassy or (liquid) crystalline phases.

\footnotetext{
${ }^{a}$ e-mail: leonard.sagis@wur.nl
} 
For such interfaces there is mounting experimental evidence that analyzing data with equation (1) may give inaccurate results (see [2] and references therein). To obtain meaningful data from tensiometry experiments on complex fluid-fluid interfaces we need a generalized form of equation (1), which also includes effects from in-plane viscous and inertial stresses, and contributions from viscous and inertial stresses exerted on the interface by the adjoining bulk phases. Javadi et al. refer to this generalized equation as the "dynamic Gauss-Laplace equation" [1].

The generalizations of the Laplace equation mentioned by Javadi et al. [1] at the end of their paper, have in fact already been derived, by researchers active in the fields of interfacial transport phenomena and nonequilibrium thermodynamics. Using Gibbs' concept of surface excess variables [3], various authors have derived a differential balance for the time rate of change of the surface excess momentum, which in the absence of in-plane viscous and inertial stresses, and negligible viscous and inertial stresses exerted on the interface by the adjoining bulk phases, reduces to the familiar Laplace equation. Scriven presented a generalized version of the Laplace equation in 1960 [4], and an improved version of this balance was published by Slattery in 1964 [5]. Bedeaux derived a momentum balance for the interface in the context of the classical irreversible thermodynamics framework (see for example reference [6] and references therein). Several textbooks are currently available that give detailed discussions of the differential surface momentum balance [7-9]. In this paper we will discuss this balance, and in particular focus on how it could be applied in the analysis of dilatational rheological experiments on complex fluid-fluid interfaces.

\section{Generalized surface momentum balances}

The Young-Laplace equation is a simplified version of the momentum balance at the interface, given by $[2,4-9]$

$$
\begin{aligned}
\rho^{s} \frac{d_{s} \mathbf{v}^{s}}{d t}= & \nabla_{s} \gamma+2 \gamma H \boldsymbol{\xi}+\nabla_{s} \cdot \boldsymbol{\sigma}^{s}+\sum_{A=1}^{N} \rho_{(A)}^{s} \mathbf{b}_{(A)}^{s} \\
& -\llbracket \rho\left(\mathbf{v}-\mathbf{v}^{s}\right)\left(\mathbf{v}-\mathbf{v}^{s}\right) \cdot \boldsymbol{\xi}+P \boldsymbol{\xi}-\boldsymbol{\sigma} \cdot \boldsymbol{\xi} \rrbracket
\end{aligned}
$$

where $\rho^{s}$ is the total surface mass density $\left(\mathrm{kg} / \mathrm{m}^{2}\right), \mathbf{v}^{s}$ is the surface velocity, $\nabla_{s}$ is the surface gradient operator [9], $\boldsymbol{\xi}$ is the surface unit normal vector, $\boldsymbol{\sigma}^{s}$ is the surface extra stress tensor, $\rho_{(A)}^{s}$ is the surface density of species $A, \mathbf{b}_{(A)}^{s}$ are the body forces acting on species $A$ at the interface, $\rho$ is the total bulk density, $\mathbf{v}$ is the bulk velocity field, and $\boldsymbol{\sigma}$ is the extra stress tensor in the bulk phase. The material derivative in (2) is defined as [9]

$$
\frac{d_{s} \psi^{s}}{d t}=\frac{\partial \psi^{s}}{\partial t}+\left(\nabla_{s} \psi^{s}\right) \cdot \dot{\mathbf{y}}
$$

where $\dot{\mathbf{y}}=\mathbf{v}^{s}-\mathbf{u}$ is the intrinsic surface velocity [9], and $\mathbf{u}$ is the speed of displacement of the interface. The boldface brackets in the second line of Eq. (2) denote contributions from the adjoining bulk phases to the balance equation, and are defined as $[9]$

$$
\llbracket \psi \boldsymbol{\xi} \rrbracket=\psi^{(I)} \boldsymbol{\xi}^{(I, J)}+\psi^{(J)} \boldsymbol{\xi}^{(J, I)}
$$

where $\psi^{(I)}$ denotes the value of an arbitrary observable $\psi$ in bulk phase $I$ (evaluated at the interface), and $\boldsymbol{\xi}^{(I, J)}$ is the unit vector normal to the interface separating phase $I$ and $J$, and pointing in the direction of phase $I$.

Equation (2) is known as either the jump momentum balance (the term we will use in the remainder of this paper) [9], the momentum interface balance [8], or the 
surface excess linear momentum balance [7], and it describes the time rate of change of the surface excess velocity as a result of surface tension gradients, in-plane viscous stresses (or viscoelastic stresses, depending on the nature of the interface), and body forces acting on the material in the interface. The boldface term denotes contributions to the time rate of change of surface momentum resulting from inertial, hydrostatic, and viscous stresses exerted on the interface by the adjoining bulk phases.

To use (2) for the analysis of dilatational data we need to supply constitutive equations for the bulk and surface extra stress tensors. For the adjoining liquid phases we will assume Newtonian behavior, and hence $\boldsymbol{\sigma}$ is given by

$$
\boldsymbol{\sigma}=\lambda(\operatorname{tr} \mathbf{D}) \mathbf{I}+2 \eta \mathbf{D}
$$

where $\eta$ is the shear viscosity of the fluid, $\mathbf{D}=\frac{1}{2}\left(\nabla \mathbf{v}+[\nabla \mathbf{v}]^{T}\right)$ is the bulk rate of deformation tensor, $\lambda=\eta_{b}-\frac{2}{3} \eta, \eta_{b}$ is the bulk viscosity of the fluid, and $\mathbf{I}$ is the three-dimensional unit tensor. When both bulk phases are incompressible fluids, the first term on the right hand side of (5) can be omitted. The constitutive equation for the extra stress tensor of the interfaces may be substantially more complex. For small deformations the extra stress tensor of viscous interfaces can be approximated by the linear Boussinesq model [4,10-12]

$$
\boldsymbol{\sigma}^{s}=\left(\varepsilon_{d}-\varepsilon_{s}\right)\left(\nabla_{s} \cdot \mathbf{v}^{s}\right) \mathbf{P}+2 \varepsilon_{s} \mathbf{D}^{s}
$$

where $\varepsilon_{d} \equiv \varepsilon_{d}\left(\rho^{s}, T^{s}, \omega_{(1)}^{s}, \ldots, \omega_{(N-1)}^{s}\right)$ is the surface dilatational viscosity, $\varepsilon_{s} \equiv \varepsilon_{s}\left(\rho^{s}\right.$, $\left.T^{s}, \omega_{(1)}^{s}, \ldots, \omega_{(N-1)}^{s}\right)$ is the surface shear viscosity, $\mathbf{P}$ is the surface projection tensor [9], and $\mathbf{D}^{s}$ is the surface rate of deformation tensor [9]. For viscoelastic interfaces a two-dimensional version of the Kelvin-Voight model is often used, given by $[2,13]$

$$
\boldsymbol{\sigma}^{s}=\left(\varepsilon_{d}-\varepsilon_{s}\right)\left(\nabla_{s} \cdot \mathbf{v}^{s}\right) \mathbf{P}+2 \varepsilon_{s} \mathbf{D}^{s}+\left(E_{d}-G_{s}\right)\left(\operatorname{tr} \mathbf{U}^{s}\right) \mathbf{P}+2 G_{s} \mathbf{U}^{s}
$$

where $G_{s} \equiv G_{s}\left(\rho^{s}, T^{s}, \omega_{(1)}^{s}, \ldots, \omega_{(N-1)}^{s}\right)$ is the surface shear modulus, and $\mathbf{U}^{s}$ is the surface displacement tensor (note that $\dot{\mathbf{U}}^{s}=\mathbf{D}^{s}$ ). Other models have been suggested for the surface stress tensor, such as the linear surface Maxwell model and surface Jeffreys model (see [2] and references therein). All these models are linear in the rate of deformation, and valid only for small deformations and deformation rates.

Apart from resistances against in-plane deformations such as shear and dilatation, complex fluid-fluid interfaces may also have a significant resistance against bending, and this resistance can also affect the dynamics of multiphase systems $[2,14-20]$. Bending rigidity is most conveniently introduced through a curvature expansion of the surface tension, given by (correct up to second order in curvature) $[21,22]$

$$
\gamma=\gamma_{0}-k C_{0} H+\bar{k} K+\frac{1}{2} k H^{2} .
$$

Here $\gamma_{0}$ is the surface tension of the flat interface, $C_{0}$ is the spontaneous curvature of the interface, $k$ is the bending rigidity of the interface associated with the mean curvature $H$, and $\bar{k}$ is the bending rigidity of the interface associated with the Gaussian curvature $K$. Incorporating this expansion in the jump momentum balance leads to (again correct up to second order in curvature) [22]

$$
\begin{aligned}
\rho^{s} \frac{d_{s} \mathbf{v}^{s}}{d t}= & \nabla_{s} \gamma_{0}-(H \mathbf{P}+\mathbf{B}) \cdot \nabla_{s}\left(k C_{0}\right)-k C_{0} \nabla_{s} H \\
& +\frac{1}{2} \boldsymbol{\xi} \nabla_{s}^{2}\left(k C_{0}\right)+2 \gamma_{0} H \boldsymbol{\xi}-k C_{0} K \boldsymbol{\xi}+\nabla_{s} \cdot \boldsymbol{\sigma}^{s} \\
& +\sum_{A=1}^{N} \rho_{(A)}^{s} \mathbf{b}_{(A)}^{s}-\llbracket \rho\left(\mathbf{v}-\mathbf{v}^{s}\right)\left(\mathbf{v}-\mathbf{v}^{s}\right) \cdot \boldsymbol{\xi}+P \boldsymbol{\xi}-\boldsymbol{\sigma} \cdot \boldsymbol{\xi} \rrbracket
\end{aligned}
$$


where $\mathbf{B}=-\nabla_{s} \boldsymbol{\xi}$ is the symmetric second groundform tangential tensor, and $\nabla_{s}^{2}$ is the surface Laplacian (defined as $\nabla_{s} \cdot \nabla_{s}$ ) [9]. We see that when bending rigidities are not negligible, the jump momentum balance takes on a much more complicated form. In the next section we will discuss when this generalized form of the surface momentum balance reduces to the familiar Laplace equation.

\section{When do the generalized balances reduce to the Young-Laplace equation?}

Let us now examine the conditions that need to be satisfied for Eq. (9) to reduce to the Young-Laplace Eq. (1). For this to happen we need to assume that in-plane inertial stresses are negligible, that stresses resulting from gradients in surface tension are negligible, that contributions from in-plane viscous stresses may be ignored, and that inertial and viscous stresses exerted on the interface by the adjoining bulk phases may be neglected. We will also need to make sure the applied deformation is uniform, to avoid gradients in surface concentration and hence avoid gradients in material properties along the interface, and avoid/minimize gradients in curvature. We will address each of these issues separately in this section.

I. In-plane inertial stresses must be negligible: for small oscillations of the droplet radius of the form $R=R_{0}+\delta R e^{i \omega t}$, the inertial term in Eq. (2) scales with the applied frequency as $\rho^{s} \omega^{2} \delta R \boldsymbol{\xi}$. Inertial effects are therefore negligible when $\rho^{s} \omega^{2} \delta R \boldsymbol{\xi}<<2 \gamma H \boldsymbol{\xi}$, or [23]

$$
\omega \ll \sqrt{\frac{2 \gamma}{\rho^{s} R \delta R}} .
$$

If we assume typical values for the parameters in this inequality, i.e. $\gamma \sim 10^{-2} \mathrm{~N} / \mathrm{m}$, $\rho^{s} \sim 10^{-6} \mathrm{~kg} / \mathrm{m}^{2}, R \sim 10^{-3} \mathrm{~m}$, and $\delta R \sim 0.1 R$, we arrive at an upper limit for the frequency $\omega$, below which inertial stresses may be neglected of the order of $10^{5} \mathrm{~s}^{-1}$. This is much higher than the frequencies which can be applied in commercially available OBM and BPT methods. But dilatational properties can also be determined using ultrasound with frequencies up to $10 \mathrm{MHz}$, and then inertial effects can no longer be neglected $[24,25]$.

II. Stresses exerted on the interface by the adjoining bulk phases must be negligible: here we will focus only on the viscous stresses exerted on the interface by the bulk phases, since inertial stresses will be relevant only at very high frequencies, upwards of $10^{5} \mathrm{~s}^{-1}$. For gas bubbles we may have contributions from the bulk viscosity term in Eq. (5). This term scales with frequency and deformation amplitude as $\lambda \omega(\delta V / V) \sim 3 \lambda \omega \delta R / R$. Comparing the magnitude of this term to the term $2 \gamma H$ in Eq. (9), we find the former is negligible when

$$
\lambda \ll \frac{\gamma}{3 \omega \delta R} .
$$

For most gasses $\lambda$ is sufficiently small that this criterion is satisfied at typical frequencies applied in tensiometry experiments. But again, in ultrasound experiments this term may not be negligible. Shear stresses will scale with frequency and deformation amplitude as $\eta \delta \mathbf{v}^{s} / R \sim \eta \omega(\delta R / R) \boldsymbol{\xi}$, and again comparing this to the term $2 \gamma H \boldsymbol{\xi}$ in Eq. (9), we find viscous shear stresses are negligible only when

$$
\eta \ll \frac{\gamma}{\omega \delta R} .
$$


If we again assume realistic values for the parameters in these expressions, i.e. $\gamma \sim$ $10^{-2} \mathrm{~N} / \mathrm{m}, R \sim 10^{-3} \mathrm{~m}$, and $\delta R \sim 0.1 R$, we arrive at an upper limit for the shear viscosity of $100 / \omega$. So for syringe based OBM systems, which have an upper limit for the frequency of at most $1 \mathrm{~Hz}$, the viscosity of the adjoining phases should be much lower than $100 \mathrm{~Pa}$ s. In most experiments this criterion will be satisfied, and viscous stresses exerted by the bulk phases can safely be ignored. Piezo driven systems have an upper limit for the frequency of about $100 \mathrm{~Hz}$, and at these frequencies the viscosities of the adjoining phases should be much less than $1 \mathrm{~Pa}$ s. Here problems may arise when studying the interfacial properties of interfaces between water and highly viscous oil phases, or glycerol and air.

When the criteria listed above are satisfied, several terms in Eq. (9) may be omitted, and the expression reduces to

$$
\begin{aligned}
& \nabla_{s} \gamma_{0}-(H \mathbf{P}+\mathbf{B}) \cdot \nabla_{s}\left(k C_{0}\right)-k C_{0} \nabla_{s} H+\frac{1}{2} \boldsymbol{\xi} \nabla_{s}^{2}\left(k C_{0}\right)+2 \gamma_{0} H \boldsymbol{\xi}-k C_{0} K \boldsymbol{\xi} \\
& \quad+\nabla_{s} \cdot \boldsymbol{\sigma}^{s}+\sum_{A=1}^{N} \rho_{(A)}^{s} \mathbf{b}_{(A)}^{s}-\llbracket P \boldsymbol{\xi} \rrbracket=0
\end{aligned}
$$

III. The applied deformation of the interface should be uniform: when deformations are non-uniform, gradients in concentration of the surface active components may develop along the interface, and hence also gradients of the material properties, such as the surface viscosities in Eq. (6) or the surface rigidity constants in Eq. (8) may develop. When material properties are a function of position on the surface, the surface momentum balance may be coupled to the species jump mass balances, given by $[9]$

$$
\rho^{s} \frac{d_{s} \omega_{(A)}^{s}}{d t}+\nabla_{s} \cdot \mathbf{j}_{(A)}^{s}-r_{(A)}^{s}+\llbracket \mathbf{j}_{(A)} \cdot \boldsymbol{\xi}+\rho\left(\omega_{(A)}-\omega_{(A)}^{s}\right)\left(\mathbf{v}-\mathbf{v}^{s}\right) \cdot \boldsymbol{\xi} \rrbracket=0
$$

where $\omega_{(A)}^{s}$ is the surface mass fraction of species $A, \mathbf{j}_{(A)}^{s}=\rho_{(A)}^{s}\left(\mathbf{v}_{(A)}^{s}-\mathbf{v}^{s}\right)$ is the surface mass flux vector, $r_{(A)}^{s}$ is the rate at which species $A$ is produced or consumed by reactions at the interface, $\mathbf{j}_{(A)}=\rho_{(A)}\left(\mathbf{v}_{(A)}-\mathbf{v}\right)$ is the mass flux vector in the adjoining bulk phases, and $\omega_{(A)}$ is the mass fraction of species $A$ in the bulk phase. Equation (14) describes the time rate of change of the surface mass fraction of component $A$ as a function of, respectively, surface diffusion, chemical reactions at the surface, and convective and diffusive transport between the bulk phase and the interface. If diffusion processes either along or perpendicular to the interface are insufficiently fast to eliminate deformation induced gradients, the surface momentum and surface mass balances will have to be solved simultaneous. Experimental evidence that this coupling can be relevant can be found elsewhere in this issue, for example, the paper by Rühs et al. [26], describing the effects of mass transfer in dilatational experiments on protein stabilized interfaces, or the paper by Sagis et al. describing deformation induced adsorption of proteins at lipid membranes [27]. Evidence of this coupling can also be found in the deformation behavior of vesicles and phase-separated biopolymer solutions $[19,20,28]$.

When the deformation is sufficiently uniform to avoid gradients in the material properties, Eq. (13) simplifies even further, to

$$
-k C_{0} \nabla_{s} H+2 \gamma_{0} H \boldsymbol{\xi}-k C_{0} K \boldsymbol{\xi}+\nabla_{s} \cdot \boldsymbol{\sigma}^{s}-\llbracket P \boldsymbol{\xi} \rrbracket=0 .
$$

In arriving at this result we have assumed that the effects of force fields acting on the material in the interfaces can also be neglected.

IV. Gradients in curvature $H$ and the bending rigidity of the interface should be small: the terms $k C_{0} \nabla_{s} H$ and $k C_{0} K \boldsymbol{\xi}$ should be negligible with respect 
to the term $2 \gamma_{0} H \boldsymbol{\xi}$. Both these terms scale with droplet radius as $k C_{0} / R^{2}$, and hence for these terms to be negligible with respect to the term $2 \gamma_{0} H \boldsymbol{\xi}$, we must have

$$
\frac{k C_{0}}{2 R}<<\gamma .
$$

When this criterion is satisfied Eq. (15) reduces to

$$
2 \gamma_{0} H \boldsymbol{\xi}+\nabla_{s} \cdot \boldsymbol{\sigma}^{s}-\llbracket P \boldsymbol{\xi} \rrbracket=0 .
$$

V. The applied deformations should be small and purely dilatational: For sufficiently small deformations we can combine the $r$-, $\theta$-, and $\varphi$-components of Eq. (17), and linearize the result, to obtain

$$
2 \gamma_{0} H+\left(\nabla_{s} \cdot \boldsymbol{\sigma}^{s}\right)_{r}+P^{(1)}-P^{(2)}=0 .
$$

If the deformations are not small or the droplet deviates significantly from a spherical shape, this linearized equation will not be sufficiently accurate. Droplets used in OBM or BPT methods should therefore always be close to spherical. The term $\left(\nabla_{s} \cdot \boldsymbol{\sigma}^{s}\right)_{r}$, equals

$$
\left(\nabla_{s} \cdot \boldsymbol{\sigma}^{s}\right)_{r}=2 H \operatorname{tr}\left[\boldsymbol{\sigma}^{s}\right] .
$$

Substituting this result in Eq. (18), we obtain

$$
2 H\left(\gamma_{0}+\operatorname{tr}\left[\boldsymbol{\sigma}^{s}\right]\right)+P^{(1)}-P^{(2)}=0 .
$$

From this we conclude that if we define a dynamic surface tension $\gamma_{d}$ as

$$
\gamma_{d} \equiv \gamma_{0}\left(\rho^{s}, T, \omega_{(1)}^{s}, \ldots, \omega_{(N-1)}^{s}\right)+\operatorname{tr}\left[\boldsymbol{\sigma}^{s}\right],
$$

we obtain the Young-Laplace Eq. (1). We see that for complex fluid-fluid interfaces the dynamic surface tension contains contributions from the actual surface tension and contributions from in-plane viscoelastic stresses. For a highly elastic interface, with an expression for the surface extra stress tensor given by the second part of Eq. (7), the dynamic surface tension is given by

$$
\gamma_{d} \equiv \gamma_{0}\left(\rho^{s}, T, \omega_{(1)}^{s}, \ldots, \omega_{(N-1)}^{s}\right)+\left(E_{d}+G_{s}\right) \operatorname{tr} \mathbf{U}^{s},
$$

and contains contributions from surface shear as well as dilatational properties. The separation of all these contributions is a nontrivial problem, which we will address elsewhere in this issue, since this is a more general problem, not restricted to OBM and BPT methods. Equation (22) was derived assuming a linear model for the surface extra stress tensor. In most studies on dilatational properties of complex fluid-fluid interfaces tests to determine whether the response of the interface to a deformation is indeed in the linear response regime are not performed. Testing outside this regime makes extracting useful information from (22) even more complicated, so as pointed out elsewhere in this issue, strain sweeps should always be a standard part of any measuring protocol for dilatational measurements on complex fluid-fluid interfaces.

\section{Conclusions and outlook}

From the above analysis we conclude that in oscillating bubble and bubble pressure tensiometry experiments on complex fluid-fluid interfaces, the Young-Laplace equation can be used for the analysis of droplet profile or pressure data only when 
all conditions I through $\mathrm{V}$ are met. A check of these conditions should be part of any standard analysis protocol. If one or more of these criteria are not met, postprocessing of the data must be performed with one of the generalized forms of the momentum balance presented here. If the applied deformations are not sufficiently uniform, the generalized momentum balances will be coupled to the species jump mass balances for the adsorbed components, and these balances will then have to be solved simultaneously. When the contributions from surface tension variations and inplane viscoelastic stresses to the dynamic surface tension are both significant, again the interfacial momentum and mass balances must be solved simultaneously. Simulation methods for multiphase systems have progressed significantly in recent years (see for example the review of numerical simulations of continuum models by Gross and Reusken [29], or the lattice Boltzmann methods described by Krüger et al. [30], elsewhere in this issue). Extracting useful data from OBM and BPT experiments for which one or more of the criteria listed above are not met may become feasible with these methods. But the computational load required to do so is still rather large and time consuming. Based on the analysis presented here we see that for fluid-fluid interfaces with adsorbed components that form complex mesophases, the determination of meaningful dilatational data with OBM and BPT methods is far from trivial, and requires a detailed and careful analysis of profile or pressure data. In view of the versatility of these methods, and accuracy problems that plague alternative methods such as Langmuir troughs, OBM and BPT methods will likely remain the main method of choice for obtaining dilatational properties. Ease of data extraction would greatly improve if smaller deformation amplitudes could be accessed $(\delta A / A<1 \%)$, and if profile or pressure analysis could somehow be combined with in situ structural analysis of the interface, which would amount to performing a two-dimensional rheooptics experiment [31]. The latter may however be difficult to realize, in view of the spherical geometry of the interface, and the fact that the location of the interface is not fixed in space.

\section{References}

1. A. Javadi, N. Mucic, M. Karbaschi, J. Won, M. Lotfi, A. Dan, V. Ulaganathan, G. Gochev, A. Makievski, V. Kovalchuk, et al., Eur. Phys. J. Special Topics 222, 7 (2013)

2. L.M.C. Sagis, Rev. Mod. Phys. 83, 1367 (2011)

3. J.W. Gibbs, The Collected Works of J. Willard Gibbs, Vol. 1 (Yale University Press, New Haven, 1928)

4. L.E. Scriven, Chem. Eng. Sci. 12, 98 (1960)

5. J.C. Slattery, Chem. Eng. Sci. 19, 379 (1964)

6. B.J.A. Zielinska, D. Bedeaux, Physica A 112, 265 (1982)

7. D.A. Edwards, H. Brenner, D.T. Wasan, Interfacial Transport Phenomena and Rheology (Butterworth-Henemann, Boston, 1991)

8. R. Gatignol, R. Prud'homme, Mechanic and Thermodynamic Modeling of Fluid Interfaces (World Scientific Publishing, Singapore, 2001)

9. J.C. Slattery, L.M.C. Sagis, E.S. Oh, Interfacial Transport Phenomena, 2nd edn. (Springer, New York, 2007)

10. J. Boussinesq, Comptes Rendus des Séances de l'Academie des Sciences 156, 983 (1913)

11. J. Boussinesq, Comptes Rendus des Séances de l'Academie des Sciences 156, 1035 (1913)

12. J. Boussinesq, Comptes Rendus des Séances de l'Academie des Sciences 156, 1124 (1913)

13. T. Verwijlen, P. Moldenaers, J. Vermant, Eur. Phys. J. Special Topics 222, 83 (2013)

14. J.B.A.F. Smeulders, C. Blom, J. Mellema, Phys. Rev. A. 42, 3483 (1990)

15. J.B.A.F. Smeulders, C. Blom, J. Mellema, Phys. Rev. A. 46, 7708 (1992)

16. K.H. de Haas, G.J. Ruiter, J. Mellema, Phys. Rev. E 52, 1891 (1995) 
17. E. Scholten, L.M.C. Sagis, E. van der Linden, J. Phys. Chem. B 108, 12164 (2004)

18. E. Scholten, L.M.C. Sagis, E. van der Linden, Macromolecules 38, 3515 (2005)

19. E. Scholten, L.M.C. Sagis, E. van der Linden, J. Phys. Chem. B 110, 3250 (2006)

20. L.M.C. Sagis, J. Controlled Release 131, 5 (2008)

21. W. Helfrich, Z. Naturforsch, C 28, 693 (1973)

22. L.M.C. Sagis, Physica A 246, 591 (1997)

23. L.M.C. Sagis, Applied Rheology 20, 24380 (2010)

24. A.A. Doinikov, J.F. Haac, P.A. Dayton, Ultrasonics 49, 263 (2009)

25. A.A. Doinikov, J.F. Haac, P.A. Dayton, Ultrasonics 49, 269 (2009)

26. P.A. Rühs, N. Scheuble, E.J. Windhab, P. Fischer, Eur. Phys. J. Special Topics 222, 47 (2013)

27. L.M.C. Sagis, E. Bijl, L. Antono, N.C.A. de Ruijter, H. van Valenberg, Eur. Phys. J. Special Topics 222, 61 (2013)

28. E. Scholten, J. Sprakel, L.M.C. Sagis, E. van der Linden, Biomacromolecules 7, 339 (2006)

29. S. Gross, A. Reusken, Eur. Phys. J. Special Topics 222, 211 (2013)

30. T. Krüger, S. Frijters, F. Günther, B. Kaoui, J. Harting, Eur. Phys. J. Special Topics 222, 177 (2013)

31. E. van der Linden, L.M.C. Sagis, P. Venema, Curr. Opinion Colloid Interface Sci. 8, 349 (2003) 